\title{
The Current Problem of Traffic Congestion on Highways
}

\author{
Yilin Jiang \\ School of North China Electric Power University, Baoding 071000, China \\ 772431198@qq.com
}

Keywords: Toll plaza, Throughput, Analytic Hierarchy Process, Queuing theory.

Abstract. In this paper, we try to designing a new toll plaza and the goal is to maximize the throughput, minimize the cost and accident times. Taking highways in New Jersey of the United States for example, the Queuing theory and Analytic Hierarchy Process are used to analyze the model.

\section{Distribute the weights of the factors by Analytic Hierarchy Process}

When designing a new toll plaza, the goal is to maximize the throughput, minimize the cost and accident times [1]. The factors which can effect the specifications are complex, so we just choose the number of tollbooths, service time, the number of waiting cars and the proportion of different type of tollbooths. In order to distribute the weights of the factors, the Analytic Hierarchy Process is introduced [2].

Analytic Hierarchy Process is to decompose decision-related elements into objectives, criteria and schemes. In this way, the factors mentioned above can be shown in figure 1 .

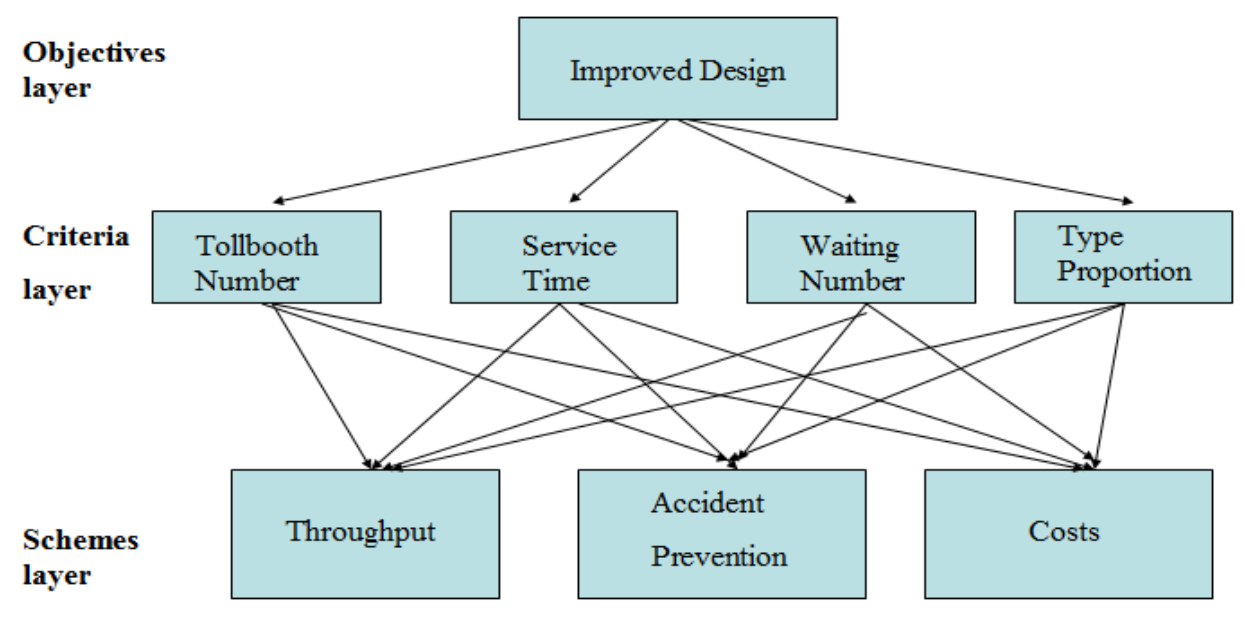

Figure 1. Progressive Hierarchy of the relative factors

The various criteria in the criteria layer do not have the same status in the measurement of the goal. They each occupy a certain proportion. Through judgmentmatrix, we can calculate each maximum eigenvalue for the matrixes.

Through the mean random consistency index, we can determine RI according to different $\mathrm{n}$. When $\mathrm{CR}<0.10$, it is considered that the consistency of the judgment matrix is acceptable. Otherwise, the judgment matrix should be modified appropriately. After testing for a few times, finally we obtain the coefficient matrix of weights for different factors as shown in table 1 and 2.

Table 1 . The weighting coefficient of the criteria layer

\begin{tabular}{|c|c|c|c|c|}
\hline Layer & $\mathrm{W}_{\mathrm{b} 1}$ & $\mathrm{~W}_{\mathrm{b} 2}$ & $\mathrm{~W}_{\mathrm{b} 3}$ & $\mathrm{~W}_{\mathrm{b} 4}$ \\
\hline $\mathrm{A}$ & 0.59 & 0.22 & 0.12 & 0.07 \\
\hline
\end{tabular}


Table 2. The weighting coefficient of the schemes layer

\begin{tabular}{|c|c|c|c|}
\hline Layer & $\mathrm{W}_{\mathrm{c} 1}$ & $\mathrm{~W}_{\mathrm{c} 2}$ & $\mathrm{~W}_{\mathrm{c} 3}$ \\
\hline $\mathrm{B}_{1}$ & 0.76 & 0.16 & 0.08 \\
\hline $\mathrm{B}_{2}$ & 0.64 & 0.26 & 0.10 \\
\hline $\mathrm{B}_{3}$ & 0.63 & 0.28 & 0.09 \\
\hline $\mathrm{B}_{4}$ & 0.67 & 0.10 & 0.23 \\
\hline
\end{tabular}

After the weighting coefficient of each layer is determined, the weight of the schemes can be calculated from that:

$$
W=\sum_{i=1}^{n} W_{a b_{i}} W_{b_{i} c_{i}}
$$

Substitute the weighting coefficients above into the formula. Then we can calculate the proportional weight.

The result is that throughput ccupies the biggest weight. The next one is accident prevention and cost is the last. As a result, throughput should be put into the first place when designing an improved toll plaza.

\section{Optimization of the throughput by queuing theory}

In order to assess the passing capability of a toll plaza, we need to calculate the largest number of cars passing through the toll plaza in an hour. And this can be reflected by throughput. In general, throughput is influenced by the number of tollbooths. As a result, the number of tollbooths should be changed.

Consider the traffic volume is the same with Model 1, in this way, according to the queuing theory, the traffic intensity $\rho$ stays unchanged. It is known that the traffic intensity of the single tollbooth ${ }^{\mu}$ can be calculated by

$$
\mu=\frac{\rho}{B}
$$

In this formula, the number of tollbooths through comparing $\mu_{\text {can }}$ be determined by looking up the standard table. It is supposed to be the best situation when the number of waiting cars is 1.0. In this way, substitute different values of B into the formula above, the actually value of $\mu$ can be obtained. Compare the value with the standard value, if the actually value is smaller, it means that the number of tollbooth can satisfy the meet of the highway. Thus, the number of tollbooth is calculated, and the value is 9 .

\section{Determine of the cost through equal proportion relationship}

The cost of a toll plaza is mainly associated with the land and road construction.

If we want to know the area of the toll plaza, we need to calculate the width of it and the length of the fan-in area first. Figure 2 is a geometric representation of the toll plaza. And the total area $\mathrm{S}$ can be calculated from that:

$$
S=S_{1}+S_{2}+S_{3}
$$




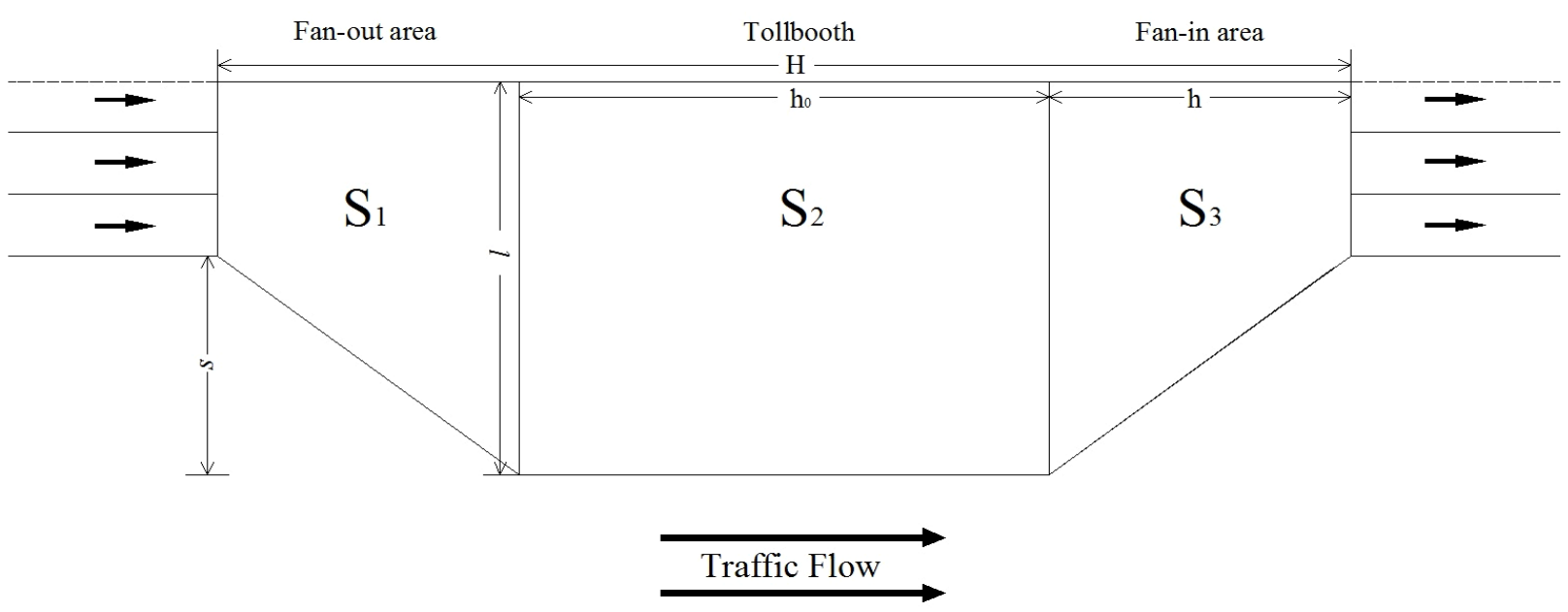

Figure 2. The geometric representation of the toll plaza

As for the width of the toll plaza $l$, it is mainly associated with the number of toll lanes $B$, lane width and tollbooth width. Moreover, lane width is dependent on the type of vehicles. Thus, we should distribute the different type of tollbooths for cars and trucks. We assume that large trucks can only pass human-staffed tollbooths, and cars can pass all types of tollbooths. In order to balance the traffic flow of each tollbooth, the distribution of the tollbooth type should satisfy that the car matches the same traffic capacity of the truck.

In order to ensure the same traffic capacity for different type of vehicle, the equal proportion relationship is introduced that

$$
\frac{R_{i} a_{i}}{m_{i}}=\frac{R_{j} a_{j}}{m_{j}}
$$

Where $R$ means occupancy rate, $a$ means the convention coefficient, $m$ means the number of tollbooth for the corresponding type. As is known that the total number of different type of tollbooths is $\mathrm{B}$, we can obtain that

$$
\sum_{i=1}^{4} m_{i}=B
$$

Through the combination of equation (18) and (19), we finally determine that the number of different types of tollbooths is that: Large trucks (human-staffed)- 3, Small cars (human -staffed) -1,Smallcars(E-ZPass)-4,Smallcars(exact-change)-1,EmergencyLine-1.Through the reference [1], we get the length of each part in a standard toll plaza as shown in table 3 .

Table 3. The standard length of each part in toll plaza

\begin{tabular}{|c|c|c|c|c|}
\hline Part & Truck lane & Car lane & Tollbooth Width & Toll island Length \\
\hline Value & $3.5 m$ & $3 m$ & $2 m$ & $36 m$ \\
\hline
\end{tabular}

By geometric relationship, the width of the toll plaza $l$ can be calculated from that

$$
l=2 B+3.5 m_{1}+3\left(B-m_{1}\right)
$$

After the length and width of the toll plaza is determined, we can obtain its area from summation of different parts. The area of different parts can be calculated by geometric area formula, and then the whole area is calculated. Through MATLAB software, the final value is obtained that the area is $16838.5 \mathrm{~m}^{2}$.

Because the cost for per unit area is viewed as constant, and the construction cost is constant, too. The total cost can be obtained from that:

$$
C=C_{0}+\alpha S
$$




\section{The graphic design of toll plaza on the flat}

Considering the weights of throughput, accident prevention and cost in the Analytic Hierarchy Process, we finally design a new toll plaza on the flat as shown in figure 3.

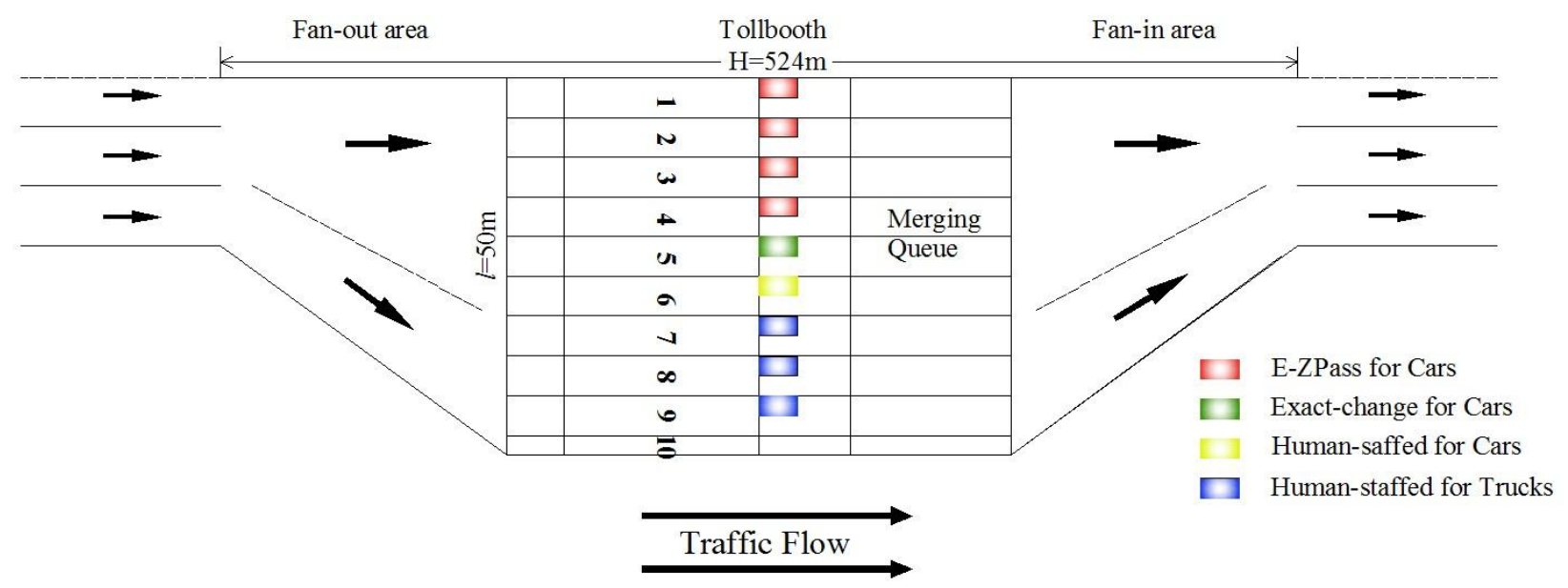

Figure 3. The graphic design of toll plaza on the flat

\section{Conclusion}

In the new design, we have calculated that throughput has increased to 2177 . The shape of the tollbooth is rectangle. The shape of fan-out area and fan-in area is the same which looks like a horn. The entire toll plaza is centrally symmetric about the central axis of the geometry. The size of it can be expressed by its area, by geometric area formula, we have obtained that the area is $16838.5 \mathrm{~m}^{2}$. And the merging pattern is showed by the arrows in figure 3 .

\section{References}

[1]Mohammad Motie and Ketan Savla, "Throughput Analysis of a Horizontal Traffic Queue under Safe Car Following Models", 2016 IEEE 55th Conference on Decision and Control (CDC), ARIA Resort \& Casino, December 12-14, 2016, Las Vegas, USA. [2]https://foursquare.com/v/bergen-toll-plaza/4b9eb66ff964a520b8fb36e3 